\title{
GENERALIZED PERIODIC RINGS
}

\author{
HOWARD E. BELL \\ Department of Mathematics \\ Brock University \\ St. Catharines \\ Ontario, Canada \\ and \\ ADIL YAQUB \\ Department of Mathematics \\ University of California \\ Santa Barbara CA, U.S.A.
}

(Received March 18, 1994 and in revised form March 13, 1995)

\begin{abstract}
Let $R$ be a ring, and let $N$ and $C$ denote the set of nilpotents and the center of $R$, respectively. $R$ is called generalized periodic if for every $x \in R \backslash(N \cup C)$, there exist distinct positive integers $m, n$ of opposite parity such that $x^{n}-x^{m} \in N \cap C$. We prove that a generalized periodic ring always has the set $\mathbf{N}$ of nilpotents forming an ideal in $\mathbf{R}$. We also consider some conditions which imply the commutativity of a generalized periodic ring.
\end{abstract}

KEY WORDS. AND PHRASES Commutativity, periodic ring, generalized periodic ring, center of a ring, commutator ideal.

1991 AMS SUBJECT CLASSIFICATION CODES. 16U80, $16 \mathrm{D} 70$.

\section{INTRODUCTION.}

Throughout the paper, $\mathbf{R}$ will denote a ring, $\mathbf{N}$ the set of nilpotents, $\mathrm{C}$ the center, $\mathrm{J}$ the Jacobson radical, and $C(R)$ the commutator ideal of $R$. The ring $R$ is called periodic if for every $x$ in $R$ there exist distinct positive integers $m, n$ such that $x^{m}=x^{n}$. An element $x$ of $R$ is called potent if, for some positive integer $n>1, x^{n}=x$. $R$ is called weakly periodic if every element $x$ of $R$ can be written as a sum of a potent element and a nilpotent element. It is well known that a periodic ring is necessarily weakly periodic. Whether a weakly periodic ring is necessarily periodic is apparently not known, except in the presence of other additional hypotheses. We now formally state the definition of a generalized periodic ring.

Definition. A ring $R$ is called generalized periodic if for every $x$ in $R, x \notin N \cup C$, we have $x^{n}-x^{m} \in N \cap C$, for some positive int egers $m, n$ of opposite parity.

Or, equivalently,

$$
x^{n}-x^{n+k} \in N \cap C ; n, k \in Z^{+} ; k \underline{\text { odd }} ;(x \notin N \cup C) \text {. }
$$


We prove that the set of nilpotents in a generalized periodic ring $R$ is always an ideal in $R$. We also consider conditions which imply the commutativity of a generalized periodic ring.

2. STRUCTURE OF GENERALIZED PERIODIC RINGS.

We begin with some basic facts about generalized periodic rings.

Lemma 1 . In a generalized periodic ring $R$, we have

(i) $\mathbf{C}(\mathbf{R}) \subseteq \mathrm{J}$;

(ii) $\mathrm{J} \subseteq \mathrm{N} \cup \mathrm{C}$;

(iii) $\mathbf{N} \subseteq \mathrm{J}$.

PROOF (i). By a well known theorem of Herstein [1], if $R$ is a division ring which satisfies (1.1), then $R$ is commutative. Next, suppose that $R$ is a primitive ring which satisfies (1.1). Since (1.1) is inherited by all subrings of $R$ and by all homomorphic images of $R$, it follows, by Jacobson's Density Theorem, that if $R$ is not a division ring, then some complete matrix ring $D_{m}$, with $m>1$, over a division ring $D$ satisfies (1.1). This, however, is false, as can be seen by taking $x=E_{12}+E_{21}$ in $D_{m}$. Hence, a primitive ring which satisfies (1.1) is necessarily a division ring, and hence is commutative by Herstein's Theorem. Therefore, any semisimple ring which satisfies (1.1) is commutative, which proves (i).

(ii). Suppose $x \in J, x \in N, x \in C$. Then, by (1.1), $x^{n}-x^{m} \in N, n \neq m$, and thus for some $q \in Z^{+}, g(\lambda) \in Z[\lambda]$

$$
x^{q}=x^{q+1} g(x) ; \quad(g(\lambda) \in Z[\lambda]) .
$$

It is readily verified that $e=[x g(x)]^{q}$ is an idempotent element in $J$ (since $x \in J$ ), and hence $e=[x g(x)]^{q}=0$. But, by $(2.1), x^{q}=x^{q} \cdot e=0$, and hence $x \in N$, contradiction. This contradiction proves (ii).

(iii). First, we prove that

$$
a x \in N \text { for all } a \in N \text { and all } x \in R \text {. }
$$

To show this, first note that by (i) and (ii),

$$
C(R) \subseteq N \cup C .
$$

Suppose (2.2) is false, and let $a \in N, x \in R, a x \notin N$ (for some a and $x$ ). Let $\bar{R}=R / C(R$ ), and let $\bar{x}=x+C(R)$ be an arbitrary element of $\bar{R}$. Since $\bar{R}$ is commutative, (2.2) implies that $\overline{a x}$ is nilpotent, and hence $(a x)^{x} \in C(R)$ for some positive integer $r$. Thus, by (2.3) $(a x)^{r} \in N$ or $(a x)^{r} \in C$. Since, by hypothesis, ax $\notin N$, therefore

$$
(a x)^{r} \in C \text { for some positive int eger } r \text {. }
$$

Since $a \in N$, let $a^{\sigma}=0$. Note that, since $(a x)^{x} \in C$,

$$
(a x)^{x}(a x)^{x}=a \cdot(a x)^{x} \cdot(x a)^{x-1} x=a^{2} x t_{1}\left(\text { some } t_{1} \in R\right) .
$$

Continuing this process, we see that

$$
\left[(a x)^{x}\right]^{k}=a^{k} k t_{k-1}\left(\text { some } t_{k-1} \in R\right) \text {, for all } k \in Z^{+} \text {. }
$$

In particular,

$$
\left[(a x)^{x}\right]^{\sigma}=a^{\sigma} x_{0-1}=0\left(\text { since } a^{\sigma}=0\right) \text {, }
$$

and hence $a x \in N$, contradiction. This contradiction proves (2.2). To complete the proof of (iii), let $a \in N, x \in R$. Then, by (2.2), ax $\in N$ and hence ax is right quasi-regular for all $x$ in $R$, which implies $a \in J$. This completes the proof of the lemma.

We are now in a position to prove the following fundamental theorem.

THEOREM 1. The set $\mathbf{N}$ of nilpotents of a generalized periodic ring $\mathbf{R}$ is an ideal of $\mathbf{R}$. 
PROOF. By Lemma 1 (iii), (ii), we have

$$
\mathrm{N} \subseteq \mathrm{J} \subseteq \mathrm{N} \cup \mathrm{C} .
$$

Let $a \in N, b \in N$. Then $a \in J, b \in J, a-b \in J$, and hence [see (2.4)] $a-b \in N$ or $a-b \in C$. If $a-b \in C$, then $a b=b a$ and hence $a-b \in N$. So, in any case, $a-b \in N$ for all $a \in N, b \in N$. We have already established [see 2.2)] that $a x \in N$ for all $a \in N, x \in R$, and a similar argument yields $x a \in N$. Therefore, $N$ is an ideal.

THEOREM 2. Let $R$ be a generalized periodic ring. Then $R / N$ is commutative, and hence $C(R) \subseteq N$.

PROOF By Theorem 1, $N$ is an ideal, and hence $R / N$ makes sense. Let $x \in R, x \notin C$. Then, by (1.1),

$$
x^{n}-x^{m} \in N \text {, for some } n>m \text {, say. }
$$

It is readily verified that

$$
\begin{aligned}
\left(x^{n-m+1}-x\right)^{m} & =\left(x^{n-m+1}-x\right) x^{m-1} g(x), \text { some } g(\lambda) \in Z[\lambda] \\
& =\left(x^{n}-x^{m}\right) g(x)
\end{aligned}
$$

and hence

$$
x^{n-m+1}-x \in N\left(\operatorname{since} x^{n}-x^{m} \in N\right) .
$$

Therefore, for all $x \in R$, we have

$$
x-x^{n-m+1} \in N \text { or } x \in C, n>m,(x \in R) .
$$

Hence, $R / N$ has the property that for each $x \in R / N$, there exists $k>1$ for which $x-x^{k}$ is central. By a well known theorem of Herstein [1], it follows that $R / N$ is commutative, and thus $C(R) \subseteq N$.

Since $N$ is an ideal of $R$ (Theorem 1), therefore $N \subseteq J$. Combining this with $C(R) \subseteq N$ and Lemma 1 (ii) we obtain

LEMMA 2. Let $R$ be a generalized periodic ring. Then

$$
C(R) \subseteq N \subseteq J \subseteq N \cup C .
$$

Next, we consider a ring which is both weakly periodic and generalized periodic.

THEOREM 3. If a ring $R$ is both generalized periodic and weakly periodic, then $R$ is periodic.

PROOF. Let $x \in R$. Since $R$ is weakly periodic, we have

$$
x=a+b \text { for some } a \in N, \quad b \text { potent }\left(b^{q}=b, q>1\right) \text {. }
$$

Thus, $x-a=(x-a)^{q}$; and since $N$ is an ideal, we have $x-x^{q} \in N$. By a well known theorem of Chacron [2], it follows that $R$ is periodic.

\section{COMMUTATIVITY OF GENERALIZED PERIODIC RINGS.}

We now turn our attention to some conditions which, when imposed on a generalized periodic ring, render it commutative. We begin with the following result, which is suggested by an old theorem on periodic rings.

THEOREM 4. Let $R$ be a generalized periodic ring, and suppose $N \subseteq C$. Then $R$ is commutative.

PROOF. By (2.6), for each $x \in R$, either $x \in C$ or $x-x^{k} \in N$ for some $k>1$. Since $N \subseteq C$, therefore, for every $x \in R, x-x^{k} \in C$ for some $k>1$. Therefore, by Herstein's Theorem [1], $R$ is commutative.

COLLARY 1. Let $R$ be a generalized periodic ring, and suppose $J \subseteq C$. Then $R$ is commutative.

PROOF. By Lemma 2, $\mathrm{N} \subseteq \mathrm{J}$, and hence $\mathrm{N} \subseteq \mathrm{C}$. Thus, $\mathrm{R}$ is commutative, by Theorem 4.

COLLARY 2. Let $R$ be a generalized periodic ring with Jacobson radical $J$. Then $J=N$ or $R$ is commutative.

PROOF. By Lemma 1 (ii), it follows that

$$
J=(J \cap N) \cup(J \cap C) \text {. }
$$


Viewing (3.1) as a relation holding on additive subgroups, we conclude that

$$
\mathrm{J}=\mathrm{J} \cap \mathrm{N} \text { or } \mathrm{J}=\mathrm{J} \cap \mathrm{C} \text {. }
$$

This implies that

$$
\mathrm{J} \subseteq \mathrm{N} \text { or } \mathrm{J} \subseteq \mathrm{C} .
$$

If $\mathrm{J} \subseteq \mathrm{N}$, then $\mathrm{J}=\mathrm{N}$ [see (2.7)]. On the other hand, if $\mathrm{J} \subseteq \mathrm{C}$, then $\mathrm{R}$ is commutative, by Collary 1 .

COROLLARY 3. Let $R$ be a generalized periodic ring which is not commutative. Then $J$ coincides with $\mathrm{N}$.

Before stating the next theorem, let us first consider the following two examples, which show that neither centrality of idempotents nor commutativity of nilpotent elements implies commutativity of a generalized periodic ring. Note that, in each case, central elements are zero divisors.

EXAMPLE 1. Let

$$
R=\left\{\left(\begin{array}{ll}
0 & 0 \\
0 & 0
\end{array}\right),\left(\begin{array}{ll}
1 & 0 \\
1 & 0
\end{array}\right),\left(\begin{array}{ll}
0 & 1 \\
0 & 1
\end{array}\right),\left(\begin{array}{ll}
1 & 1 \\
1 & 1
\end{array}\right) \mid 0,1 \in \mathrm{GF}(2)\right\} .
$$

It is readily verified that $R$ is a generalized periodic ring with commuting nilpotents but its idempotents are not in the center.

EXAMPLE 2. Let

$$
R=\left\{\left(\begin{array}{ccc}
0 & a & b \\
0 & 0 & c \\
0 & 0 & 0
\end{array}\right) \mid a, b, c, \in G F(3)\right\} .
$$

It can be seen that $R$ is, again a generalized periodic ring with central idempotents but its nilpotents do not commute with each other.

Experience shows that a condition which does not imply commutativity for general rings may do so for rings with 1 . Indeed, we can show that generalized periodic rings with 1 are commutative; in fact, in the following theorem, we can do better than that.

THEOREM 5. Suppose that $R$ is a generalized periodic ring containing a central element which is not a zero divisor. Then $R$ is commutative.

PROOF. In view of Theorem 4 , we need only show that $\mathrm{N} \subseteq \mathrm{C}$. Suppose not, and choose $a_{0} \in N \backslash C$. Let $\sigma_{0}>1$ be the minimal positive integer for which $a_{0}^{\sigma} \in C$ for all $\sigma \geq \sigma_{0}$; and let $a=a_{0}^{\sigma_{0}-1}$. Note that $a \notin C$, and $a^{\lambda} \in C$ for all $\lambda \geq 2$. Now if $c \in C$ is not a zero divisor, then $c+a \notin N \cup C$, so there exist $n, m$ of opposite parity with $n>m$, such that

$$
(c+a)^{n}-(c+a)^{m} \in N \cap C,(n>m) \text {. }
$$

We shall assume that $\mathrm{n}$ is even and $\mathrm{m}$ is odd, the other case being only marginally different.

From (3.4) we have $n c^{n-1} a-m c^{m-1} a \in C$, from which it follows that (since $c$ is not a zero divisor)

$$
n c^{n-m} a-m a \in C .
$$

Another consequence of (3.4) is that $c^{n}-c^{m} \in N$ and hence $c^{j}-c \in N$, where $j$ is the even integer $n-m+1$. Replacing $c$ by $-c$ in our argument, we also get an even integer $k$ such that $(-c)^{k}-(-c) \in N$. Since $N$ is an ideal, we have $c^{1+s(j-1)}-c \in N$ and $(-c)^{1+1(k-1)}-(-c) \in N$ for all positive integers $s$ and $t$; and taking $q=1+(j-1)(k-1)$, we see that $q$ is even, $c^{q}-c \in N$ and $(-c)^{q}-(-c) \in N$. It follows at once that $2 c \in N$ and hence $2^{r} c^{r}=0$ for some positive integer $r$. Since $c$ is not a zero divisor, this yields $2^{r} R=\{0\}$; and, in particular,

$$
2^{r} \mathrm{a} \in \mathrm{C} \text {. }
$$

By hypothesis, $n$ is even, say $n=2 n_{0}$, and hence (3.5) yields 


$$
\mathrm{ma}=2 \mathrm{n}_{0} \mathrm{c}^{\mathrm{n}-\mathrm{m}} \mathrm{a}+\mathrm{z}_{1}, \quad \mathrm{z}_{1} \in \mathrm{C} .
$$

Therefore, using (3.7) we see that

$$
\begin{aligned}
m^{2} a & =2 n_{0} c^{n-m} m a+m z_{1} \\
& =2 n_{0} c^{n-m}\left(2 n_{0} c^{n-m} a+z_{1}\right)+m z_{1} \\
& =2^{2}\left(n_{0} c^{n-m}\right)^{2} a+z_{2}, \quad z_{2} \in C ;
\end{aligned}
$$

and proceeding inductively, we get (see (3.6))

$$
\mathrm{m}^{\mathrm{r}} \mathrm{a} \in \mathrm{C} .
$$

Since $m$ was odd, (3.6) and (3.8) are incompatible with the assumption that $a \notin C$. Therefore $N \subseteq C$, as required. This proves the theorem.

COLLARY 4. Let $R$ be a ring with 1 . If $R$ is generalized periodic, then $R$ is commutative.

COLLARY 5. Let $R$ be a prime ring with nonzero center. If $R$ is generalized periodic, then $R$ is commutative.

Our final theorem confronts the impediments of Examples 1 and 2 in a more direct way.

THEOREM 6. Suppos $R$ is a generalized periodic ring, $N$ the set of nilpotents, and $E$ the set of idempotents of $R$. Suppose that

(i) $\mathrm{E} \subseteq \mathrm{C}$ (center of $\mathrm{R}$ ); and

(ii) Every commutator $[a, b]=a b-b a$ with $a \in N$ and $b \in N$ is potent

$$
\text { (i.e., }[a, b]^{q}=[a, b] \text { for some } q>1 \text { ). }
$$

Then $R$ is commutative.

PROOF. By (2.7), $C(R) \subseteq N$, and hence $[a, b] \in N$. By hyothesis, $[a, b]=[a, b]^{q}=[a, b]^{1+\lambda(q-1)}$ for all positive integers $\lambda$, and hence $[a, b]=0($ since $[a, b] \in N)$. Thus,

$$
[a, b]=0 \text { for all } a, b \in N \text { i.e., } N \text { is commutative. }
$$

Recall also that, in (2.6), we proved that, for ever $\mathrm{x}$ in $\mathrm{R}$, we have

$$
x-x^{k} \in N \text { for some } k>1 \text {, or } x \in C, \quad(x \in R) \text {. }
$$

Combining (3.9), (3.10), we see that

$$
\text { For all } x, y \text { in } R,\left[x-x^{k}, y-y^{r}\right]=0 \text { for some } k>1, r>1 \text {. }
$$

As is well known,

$$
R \cong a \text { subdirect sum of subdirectly irreducible rings } R_{1}(i \in \Gamma) \text {. }
$$

We now take a closer look at the structure of each of these subdirect summands $R_{i}$, with an eye towards proving their commutativity.

CASE 1: $\mathbf{R}_{\mathbf{i}}$ does not have an identity.

Let $\sigma: R \rightarrow R_{i}$ be the natural homomorphism of $R$ onto $R_{i}$, and let $\sigma: x \rightarrow x_{1}$. Let $N_{i}$ and $C_{i}$ denote the set of nilpotents and the center of $R_{i}$, respectively. We claim that

$$
R_{i} \subseteq N_{1} \cup C_{i} .
$$

Suppose not. Let $x_{i} \in R_{i}, x_{i} \notin N_{i}, x_{i} \notin C_{i}$, and let $\sigma: x \rightarrow x_{i},(x \in R)$. Then, clearly, $x \notin N$ and $x \notin C$, and hence by $(1.1)$,

$$
x^{n}-x^{m} \in N \text { for some positive integers } n \text { and } m, n \neq m \text {. }
$$

This implies (see the proof of Lemma 1 (ii)) that

$$
x^{q}=x^{q} e \text { for some positive integer } q \text { and some idempotent } e \text { in } R \text {. }
$$


By hypothesis (i), e is a central idempotent, and hence

$$
x^{q}=x^{9} e, e^{2}=e \in C .
$$

This implies, in $R_{1}$, that

$$
x_{1}^{q}=x_{1}^{q} e_{1}, e_{1}^{2}=e_{1} \in C_{1} .
$$

Since $e_{1}$ is a central idempotent in the subdirectly irreducible ring $R_{1}$, therefore $e_{1}=0$ (recall that $R_{1}$ does not have an identity), and hence by (3.14), $x_{1}^{q}=0$, a contradiction, since $x_{1}$ is not nilpotent. This contradiction proves (3.13).

Returning to (3.11), we see that

$$
\left[\mathrm{x}_{1}-\mathrm{x}_{1}^{\mathrm{k}}, \mathrm{y}_{1}-\mathrm{y}_{1}^{\mathrm{r}}\right]=0 ; \mathrm{k}>1, \mathrm{r}>1 ; \mathrm{x}_{1}, \mathrm{y}_{1} \in \mathrm{R}_{1} \text { (arbitrary). }
$$

Now, by a trivial minimality argument, it is readily verified tht (3.15) implies:

$$
\left[a_{i}, b_{i}\right]=0 \text { for all nilpotents } a_{i}, b_{1} \text { in } R_{1} \text {; (i.e., } N_{1} \text { is commutative). }
$$

Combining (31.13) and (3.16), we see tha $R_{i}$ is commutative.

CASE 2: $\mathbf{R}_{\mathrm{i}}$ has an identity.

Since the homomorphic image of a generalized periodic ring is also generalized periodic, it follows that $R_{i}$ is commutative, by Corollary 4 .

- Since each $\mathbf{R}_{\mathbf{i}}$ in the subdirect sum representation (3.12) is commutative, therefore the ground ring $\mathbf{R}$ itself is also commutative, and the theorem is proved.

COLLARY 6. Any generalized periodic ring with central idempotents and commuting nilpotents is commutative.

\section{REFERENCES}

1. HERSTEIN, I. N., A generalization of a theorem of Jacobon III, Amer. J. Math. 75 (1953), 105111.

2. CHACRON, M., On a theorem of Herstein, Canad. L. Math. 21 (1969), 1348-1353. 


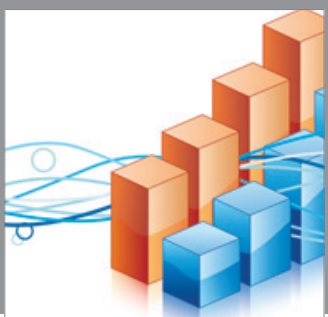

Advances in

Operations Research

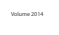

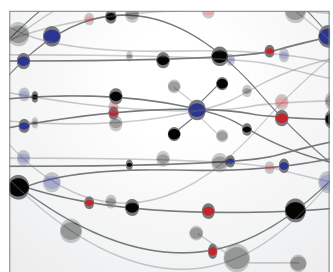

\section{The Scientific} World Journal
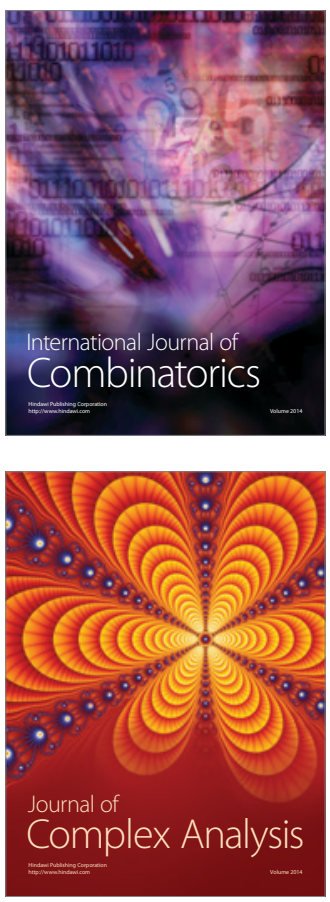

International Journal of

Mathematics and

Mathematical

Sciences
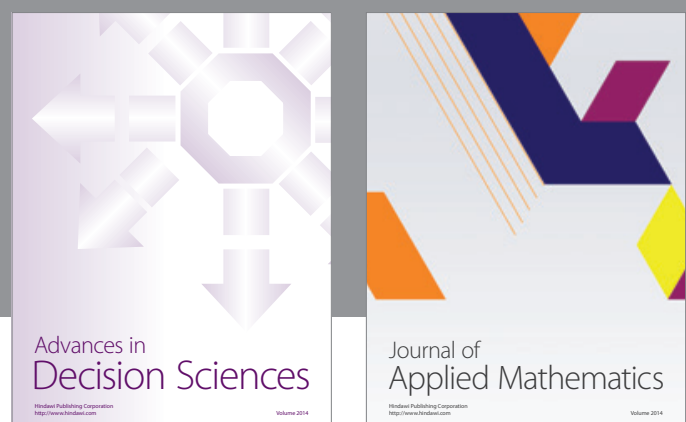

Journal of

Applied Mathematics
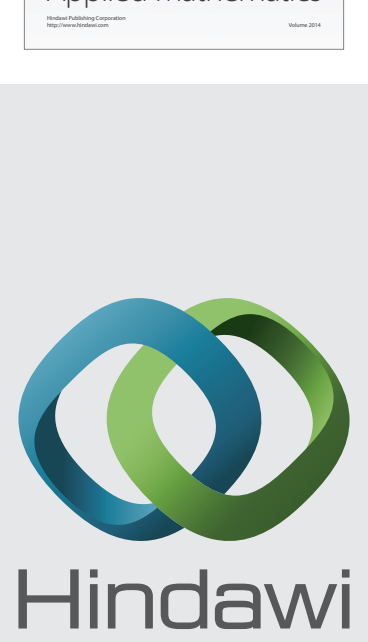

Submit your manuscripts at http://www.hindawi.com
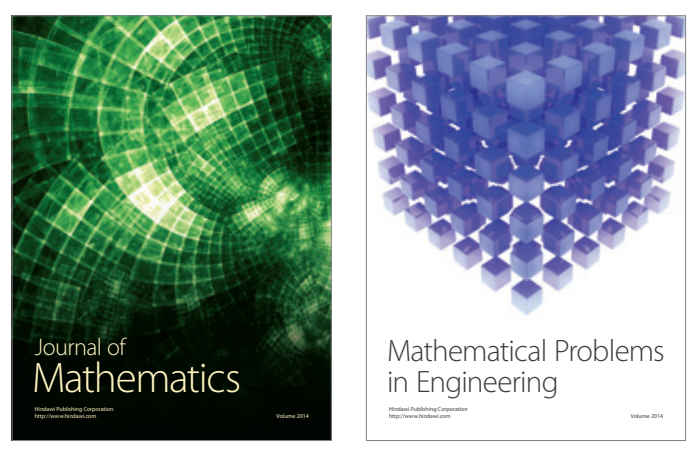

Mathematical Problems in Engineering
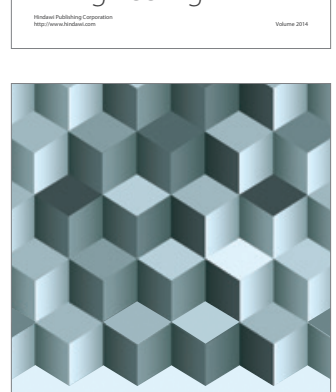

Journal of

Function Spaces
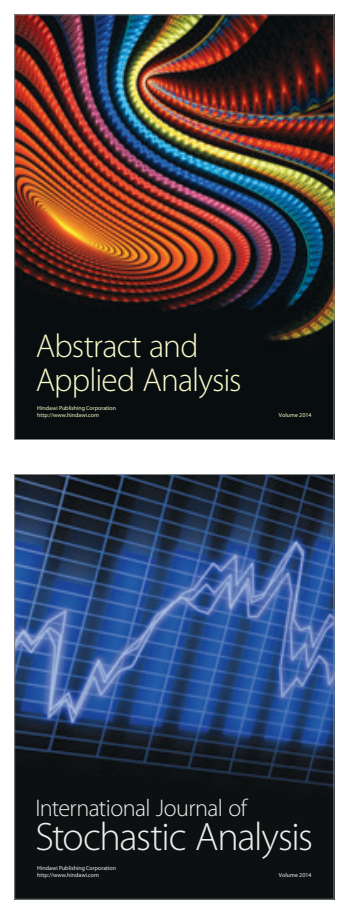

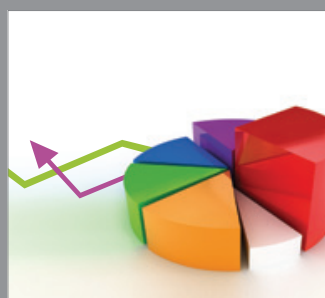

ournal of

Probability and Statistics

Promensencen
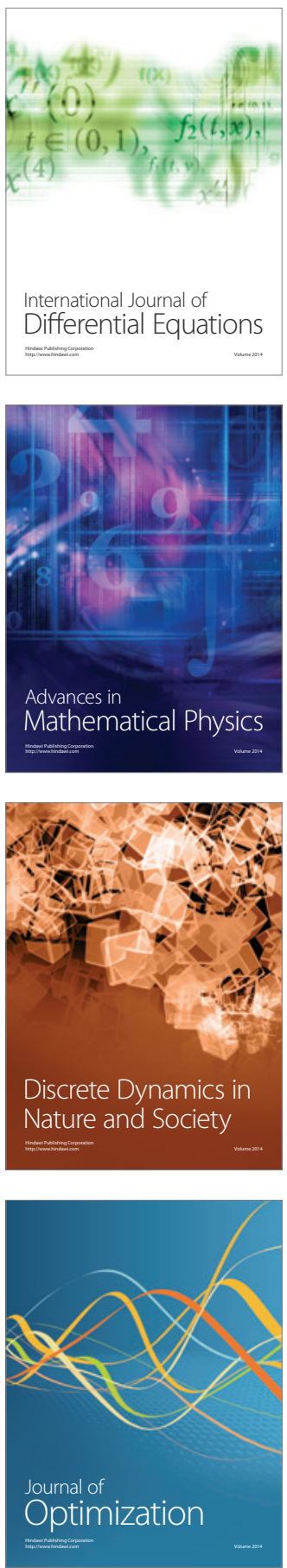